\title{
ON THE VOLUME OF A SMALL EXTRINSIC BALL IN A HYPERSURFACE OF THE HYPERBOLIC SPACE
}

\author{
FRANCISCO J. CARRERAS*, AND ANTONIO M. NAVEIRA*
}

\begin{abstract}
.
We give a power series formula for the volume of a small extrinsic geodesic ball of a hypersurface of the hyperbolic space and derive some geometric consequences.
\end{abstract}

\section{Introduction.}

Let $M$ be a $k$-dimensional Riemannian manifold. A problem to which a lot of work has been devoted is that of finding to what extent does the volume of small geodesic balls determine the geometry of $M$. In this context, a remarkable paper is [GV]. A variation of this problem have also been considered. If we restrict ourselves to submanifolds $M$ of the euclidean space $\mathrm{R}^{n+1}$, then, instead of considering the volume of (intrinsic) geodesic balls of $M$ we can consider the volumes of the intersections with $M$ of geodesic balls of $\mathrm{R}^{n+1}$ (extrinsic geodesic balls of $M$ ). The question then is the following: If we assume that the volume of every extrinsic ball in $M$, of sufficiently small radius, coincides with the volume of the ball of the same radius in $\mathrm{R}^{k}$, what can be said about the submanifold $M$ ? This problem has been dealt with in [KP], for hypersurfaces of the euclidean space, and in [KaP], for submanifolds of codimension greater than 1. (In [KP] the authors also determine those submanifolds of $\mathrm{R}^{n+1}$ which satisfy the condition on the volume of extrinsic balls for all radii.)

We consider here the problem for hypersurfaces of the hyperbolic space $\mathrm{H}^{n+1}$, instead of $\mathrm{R}^{n+1}$. To begin with, we prove the following power series formula for the volume of an extrinsic ball in a hypersurface of $\mathrm{H}^{n+1}$ :

THEOREM 1.1 Let $M \subset \mathrm{H}^{n+1}$ be a hypersurface of class $C^{5}$. Then, for any $m \in M$ and any sufficiently small $r$, we have the following Taylor formula:

\footnotetext{
* Work partially supported by a DGICYT Grant No. PB94-0972 and by E.C. Contract CHRX-CT92-0050 "GADGET II".

Received July 29, 1996.
} 


$$
\operatorname{vol}\left(B^{n+1}(m, r) \cap M\right)=\alpha_{n} r^{n}\left(1+\frac{A(m) r^{2}}{24(n+2)}+\frac{B(m) r^{4}}{5760(n+2)(n+4)}+O\left(r^{5}\right)\right),
$$

where $B^{n+1}(m, r)$ is the $(n+1)$-dimensional open ball with centre $m \in M$ and radius $r>0$ in $\mathrm{H}^{n+1}, \alpha_{n}$ is the volume of the unit ball in $\mathrm{R}^{n}$, vol means $n$-dimensional volume, and $A$ and $B$ are functions on $M$ given by

$$
\left\{\begin{aligned}
A= & 6\|\vec{h}\|^{2}-3 h^{2}+4 n(n-1), \\
B= & 1440\langle\Delta \vec{h}, \vec{h}\rangle+960\|\nabla \vec{h}\|^{2}+180\|R\|^{2}+360\|\rho\|^{2}-360 h \Delta h \\
& -120\left(n^{2}-6 n+14\right) h^{2}+45 h^{4}-240\left(n^{2}+2\right)\|\vec{h}\|^{2}-540 h^{2}\|\vec{h}\|^{2} \\
& +900\|\vec{h}\|^{4}+8 n(n-1)\left(10 n^{2}-39 n+62\right) .
\end{aligned}\right.
$$

In the above theorem, and throughout this paper, $\vec{h}$ denotes the second fundamental form of $M$, and $h=\operatorname{trace} \vec{h}$ is the (non-averaged) mean curvature of $M . \nabla$ is the Levi-Civita connection on $M, R$, the Riemann curvature tensor, and $\rho$ the Ricci curvature tensor. $\Delta$ denotes the "rough" Laplacian, that is, if $T$ is a tensor field on $M, \Delta T$ is the trace of $\nabla^{2} T$.

Next, we consider the condition that the extrinsic balls of $M$ have the same volume as the corresponding balls in the hyperbolic space. To be more precise, we say that

"A $k$-dimensional submanifold $M$ of $\mathrm{H}^{n+1}$ satisfies the Hyperbolic Extrinsic Volume Condition (HEVC) if $\operatorname{vol}\left(B^{n+1}(m, r) \cap M\right)=$ the volume of the geodesic ball of radius $r$ in $\mathrm{H}^{k}$, for every $m \in M$ and sufficiently small $r$."

Then, we prove the following results:

Proposition 1.2 a) A connected hypersurface $M^{1} \subset \mathrm{H}^{2}$ satisfies HEVC $\Longleftrightarrow M^{1}$ is an open geodesic segment.

b) A connected hypersurface $M^{2} \subset \mathrm{H}^{3}$ satisfies $\mathrm{HEVC} \Longleftrightarrow M^{2}$ is totally umbilical.

Proposition $1.3 M^{n} \subset \mathrm{H}^{n+1}$ satisfies $\mathrm{HEVC} \Longrightarrow$

i) $\tau+n(n+1) \geq 0$.

ii) $\tau=-n(n+1)$ or $M$ is minimal $\Longrightarrow M$ is totally geodesic.

iii) $M$ cannot be umbilical, except for $n=2$.

Proposition 1.4 Let $M^{3} \subset \mathrm{H}^{4}$ be connected, with $\nabla \vec{h}=0$. Then $M$ satisfies $\mathrm{HEVC}$ if and only if it is totally geodesic.

This shows that the case of the hyperbolic space is different from that of the Euclidean space: under the same hypotheses, a connected hypersurface of $\mathrm{R}^{4}$ with parallel second fundamental form, satisfying the extrinsic volume condition must be totally geodesic or an open subset of $S^{2}(r) \times \mathrm{R}[\mathrm{KP}]$. 


\section{Computation of the riemannian invariants.}

Let us denote by $\mathrm{H}^{n+1}$ the $(n+1)$-dimensional hyperbolic space of constant sectional curvature -1 , represented by Poincaré half-plane; that is,

$$
\mathrm{H}^{n+1}=\left\{\left(x_{0}, x_{1}, \cdots, x_{n}\right) \in \mathrm{R}^{n+1} \text { such that } x_{0}>0\right\},
$$

with the metric

$$
g=\frac{1}{x_{0}^{2}} \sum_{i=0}^{n} d x_{i}^{2}
$$

In what follows we shall denote the coordinate $x_{0}$ by $z$, and $\partial_{0}, \cdots, \partial_{n}$ will denote a global reference of vector fields in $\mathrm{H}^{n+1}$. Let $M$ be a topologically embedded hypersurface, let $m$ be a point in $M$, and let us take the coordinates $z, x_{1}, \cdots, x_{n}$ in $\mathrm{H}^{n+1}$ so that $m=(1,0, \cdots, 0)$, the $z$-axis is orthogonal to $M$ at $m$, and the $x_{i}$-axes, $1 \leq i \leq n$, lie in the principal directions at $m$. Then, $\left(x_{1}, \cdots, x_{n}\right)$ are local coordinates of $M$ in a neighbourhood of $m$ and, near $m$, the hypersurface $M$ can be expressed as a graph

$$
\vec{M}\left(x_{1}, \cdots, x_{n}\right)=\left(z\left(x_{1}, \cdots, x_{n}\right), x_{1}, \cdots, x_{n}\right),
$$

where $\left(x_{1}, \cdots, x_{n}\right)$ lies in a neighbourhood of the origin of $\mathrm{R}^{n}$.

Near the origin, we can write

$$
z=1+\frac{1}{2} \sum\left(\lambda_{A}-1\right) x_{A}^{2}+\frac{1}{6} \sum K_{A B C} x_{A} x_{B} x_{C}+\frac{1}{24} \sum L_{A B C D} x_{A} x_{B} x_{C} x_{D}+O\left(\rho^{5}\right)
$$

where $\lambda_{1}, \cdots, \lambda_{n}$ are the principal curvatures at $m$,

$$
\rho=\left(\sum_{A=1}^{n} x_{A}^{2}\right)^{1 / 2}
$$

and the summation in (2.2) extends to all repeating indices from 1 to $n$. (The same summation convention is used throughout this section.) It follows easily that

$$
\begin{aligned}
\frac{1}{z}=1 & -\frac{1}{2} \sum\left(\lambda_{A}-1\right) x_{A}^{2}-\frac{1}{6} \sum K_{A B C} x_{A} x_{B} x_{C}+\frac{1}{4}\left(\sum\left(\lambda_{A}-1\right) x_{A}^{2}\right)^{2} \\
& -\frac{1}{24} \sum L_{A B C D} x_{A} x_{B} x_{C} x_{D}+O\left(\rho^{5}\right)
\end{aligned}
$$

and 
ON THE VOLUME OF A SMALL EXTRINSIC BALL IN A HYPERSURFACE ... 223

$$
\begin{aligned}
\frac{1}{z^{2}}= & -\sum\left(\lambda_{A}-1\right) x_{A}^{2}-\frac{1}{3} \sum K_{A B C} x_{A} x_{B} x_{C}+\frac{3}{4}\left(\sum\left(\lambda_{A}-1\right) x_{A}^{2}\right)^{2} \\
& -\frac{1}{12} \sum L_{A B C D} x_{A} x_{B} x_{C} x_{D}+O\left(\rho^{5}\right)
\end{aligned}
$$

We get a basis of the tangent space of $M$ at $m$ by taking partial derivatives in (2.1):

$$
\vec{M}_{A}=\left(z_{A}^{\prime}, 0, \cdots, 1, \cdots, 0\right), \quad A=1, \cdots, n .
$$

With respect to this basis, the coefficients of the Riemann metric of $M$, near $m$, are given by

$$
g_{A B}=\left\langle\vec{M}_{A}, \vec{M}_{B}\right\rangle=\frac{1}{z^{2}}\left(\delta_{A B}+z_{A}^{\prime} z_{B}^{\prime}\right)
$$

we, further, choose as a unit normal vector field

$$
\nu=z D^{-1 / 2}\left(-\partial_{0}+\sum_{A=1}^{n} z_{A}^{\prime} \partial_{A}\right),
$$

where

$$
D=1+\sum_{A=1}^{n} z_{A}^{\prime 2} .
$$

With respect to this normal vector field, the coefficients of the second fundamental form are given by

$$
h_{A B}=h\left(M_{A}, M_{B}\right)=\frac{1}{z^{2}} D^{-1 / 2}\left(\delta_{A B}+z z_{A B}^{\prime \prime}+z_{A}^{\prime} z_{B}^{\prime}\right) .
$$

From (2.2) we have

$$
z_{A}^{\prime}=\left(\lambda_{A}-1\right) x_{A}+\frac{1}{2} \sum_{B, C} K_{A B C} x_{B} x_{C}+O\left(\rho^{3}\right)
$$

and

$$
z_{A B}^{\prime \prime}=\left(\lambda_{A}-1\right) \delta_{A B}+\sum_{C} K_{A B C} x_{C}+\frac{1}{2} \sum_{C, D} L_{A B C D} x_{A} x_{B} x_{C} x_{D}+O\left(\rho^{3}\right)
$$

Differentiating in (2.6), we find

$$
\frac{\partial g_{A B}}{\partial x_{C}}=\frac{1}{z^{2}}\left(z_{A C}^{\prime \prime} z_{B}^{\prime}+z_{A}^{\prime} z_{B C}^{\prime \prime}\right)-\frac{2}{z^{3}} z_{C}^{\prime}\left(\delta_{A B}+z_{A}^{\prime} z_{B}^{\prime}\right),
$$

and 


$$
\begin{aligned}
\frac{\partial^{2} g_{A B}}{\partial x_{C} \partial x_{D}}= & \frac{1}{z^{2}}\left(z_{A C D}^{\prime \prime \prime} z_{B}^{\prime}+z_{A}^{\prime} z_{B C D}^{\prime \prime \prime}+z_{A C}^{\prime \prime} z_{B D}^{\prime \prime}+z_{A D}^{\prime \prime} z_{B C}^{\prime \prime}\right) \\
& -\frac{2}{z^{3}} z_{D}^{\prime}\left(z_{A C}^{\prime \prime} z_{B}^{\prime}+z_{A}^{\prime} z_{B C}^{\prime \prime}\right)-\frac{2}{z^{3}} z_{C}^{\prime}\left(z_{A D}^{\prime \prime} z_{B}^{\prime}+z_{A}^{\prime} z_{B D}^{\prime \prime}\right) \\
& -\frac{2}{z^{3}} z_{C D}^{\prime \prime}\left(\delta_{A B}+z_{A}^{\prime} z_{B}^{\prime}\right)+\frac{6}{z^{4}} z_{C}^{\prime} z_{D}^{\prime}\left(\delta_{A B}+z_{A}^{\prime} z_{B}^{\prime}\right) .
\end{aligned}
$$

In particular,

$$
g_{A B}(m)=\delta_{A B}, \quad \frac{\partial g_{A B}}{\partial x_{C}}(m)=0,
$$

and

$$
\frac{\partial^{2} g_{A B}}{\partial x_{C} \partial x_{D}}(m)=\left(\delta_{A C} \delta_{B D}+\delta_{A D} \delta_{B C}\right)\left(\lambda_{A}-1\right)\left(\lambda_{B}-1\right)-2 \delta_{C D} \delta_{A B}\left(\lambda_{C}-1\right)
$$

From the classical formula for the Christoffel symbols ([KN]), we get

$$
\Gamma_{A B}^{C}(m)=0, \quad 1 \leq A, B, C \leq n .
$$

and

$$
\begin{aligned}
& \frac{\partial \Gamma_{A A}^{C}}{\partial x_{C}}(m)=\lambda_{A}\left(\lambda_{C}-1\right), \quad A \neq C \\
& \frac{\partial \Gamma_{A A}^{A}}{\partial x_{A}}(m)=\left(\lambda_{A}-1\right)\left(\lambda_{A}-2\right) \\
& \frac{\partial \Gamma_{A B}^{A}}{\partial x_{B}}(m)=-\left(\lambda_{B}-1\right), \quad A \neq B \\
& \frac{\partial \Gamma_{A B}^{C}}{\partial x_{D}}(m)=0, \text { if at least three of the indices } A, B, C, D \text { are different. }
\end{aligned}
$$

Let us now consider the components of the curvature tensor, defined as in [KN], given by

$$
R\left(\partial_{C}, \partial_{D}\right) \partial_{B}=\sum R_{B C D}^{A} \partial_{A}, \quad R_{A B C D}=\sum g^{A F} R_{B C D}^{F}
$$

Then,

$$
R_{B C D}^{A}=\frac{\partial \Gamma_{D B}^{A}}{\partial x_{C}}-\frac{\partial \Gamma_{C B}^{A}}{\partial x_{D}}+\sum\left(\Gamma_{D B}^{F} \Gamma_{C F}^{A}-\Gamma_{C B}^{F} \Gamma_{D F}^{A}\right)
$$

and from equations (2.16) and (2.17), we get

$$
R_{A B A B}(m)=\lambda_{A} \lambda_{B}-1, \quad A \neq B
$$

$R_{A B C D}(m)=0, \quad$ if there are three different indices among $A, B, C, D$. 
Since the $\vec{M}_{A}, 1 \leq A \leq n$ are an orthonormal basis at $m$, we get

$$
\begin{aligned}
\|R\|^{2}(m)= & 2 \sum_{A \neq B}\left(\lambda_{A} \lambda_{B}-1\right)^{2}=2\left(\left(\sum_{A=1}^{n} \lambda_{A}^{2}\right)^{2}-\sum_{A=1}^{n} \lambda_{A}^{4}\right) \\
& +4\left(\left(\sum_{A=1}^{n} \lambda_{A}^{2}\right)-\left(\sum_{A=1}^{n} \lambda_{A}\right)^{2}\right)+2 n(n-1) \\
= & 2\left(\left(\sum_{A=1}^{n} \lambda_{A}^{2}\right)^{2}-\left(\sum_{A=1}^{n} \lambda_{A}^{4}\right)+2\left(\sum_{A=1}^{n} \lambda_{A}^{2}\right)-2\left(\sum_{A=1}^{n} \lambda_{A}\right)^{2}\right)+2 n(n-1) .
\end{aligned}
$$

From (2.9), we get

$$
h_{A A}(m)=\lambda_{A}, \quad h_{A B}(m)=0, \text { if } A \neq B, \quad 1 \leq A, B \leq n,
$$

whence

$$
h(m)=\sum_{A=1}^{n} \lambda_{A}, \quad\|\vec{h}\|^{2}(m)=\sum_{A=1}^{n} \lambda_{A}^{2} .
$$

This allows us to express the curvature invariants of order 2 as follows

$$
\|R\|^{2}(m)=2\left(\|\vec{h}\|^{4}-\sum_{A=1}^{n} \lambda_{A}^{4}+2\|\vec{h}\|^{2}-2 h^{2}\right)(m)+2 n(n-1),
$$

$$
\begin{aligned}
\|\rho\|^{2}(m) & =\sum_{A=1}^{n} \rho_{A A}^{2}=\sum_{A=1}^{n}\left(\sum_{B=1}^{n} R_{A B A B}\right)^{2}=\sum_{A=1}^{n}\left(h \lambda_{A}-\lambda_{A}^{2}-(n-1)\right)^{2}(m) \\
& =\left(h^{2}\|\vec{h}\|^{2}+\sum_{A=1}^{n} \lambda_{A}^{4}+n(n-1)^{2}-2 h \sum_{A=1}^{n} \lambda_{A}^{3}-2(n-1) h^{2}+2(n-1)\|\vec{h}\|^{2}\right)(m)
\end{aligned}
$$

and the scalar curvature

$$
\tau(m)=\sum_{A=1}^{n} \rho_{A A}=\sum_{A=1}^{n}\left(h \lambda_{A}-\lambda_{A}^{2}-(n-1)\right)(m)=\left(h^{2}-\|\vec{h}\|^{2}-n(n-1)\right)(m) .
$$

Using (2.10) we get

$$
D^{-1 / 2}=1-\frac{1}{2} \sum_{A=1}^{n} z_{A}^{\prime 2}+\cdots=1-\frac{1}{2} \sum_{A=1}^{n}\left(\lambda_{A}-1\right)^{2} x_{A}^{2}+O\left(\rho^{3}\right),
$$

and from this expression, (2.10) and (2.11) we find 


$$
\begin{aligned}
h_{A B}= & \left(1-\sum_{C=1}^{n}\left(\lambda_{C}-1\right) x_{C}^{2}+O\left(\rho^{3}\right)\right)\left(1-\frac{1}{2} \sum_{C=1}^{n}\left(\lambda_{C}-1\right)^{2} x_{C}^{2}+O\left(\rho^{3}\right)\right) \\
& \left(\delta_{A B}+\left(1+\frac{1}{2} \sum_{C}\left(\lambda_{C}-1\right) x_{C}^{2}+O\left(\rho^{3}\right)\right)\left(\left(\lambda_{A}-1\right) \delta_{A B}+\sum_{C} K_{A B C} x_{C}+\right.\right. \\
& \left.\frac{1}{2} \sum_{C, D} L_{A B C D} x_{C} x_{D}+O\left(\rho^{3}\right)\right)+\left(\left(\lambda_{A}-1\right) x_{A}+\sum K_{A B C} x_{B} x_{C}+O\left(\rho^{3}\right)\right) . \\
& \left.\left(\left(\lambda_{B}-1\right) x_{B}+\sum K_{A B C} x_{A} x_{C}+O\left(\rho^{3}\right)\right)\right) \\
= & \lambda_{A} \delta_{A B}+\sum K_{A B C} x_{C}+\frac{1}{2} \sum L_{A B C D} x_{C} x_{D}+\frac{1}{2}\left(\lambda_{A}-1\right) \delta_{A B} \sum\left(\lambda_{C}-1\right) x_{C}^{2} \\
& +\left(\lambda_{A}-1\right)\left(\lambda_{B}-1\right) x_{A} x_{B}-\frac{1}{2} \lambda_{A} \delta_{A B} \sum\left(\lambda_{C}^{2}-1\right) x_{C}^{2}+O\left(\rho^{3}\right) .
\end{aligned}
$$

(Observe that from this expression it follows that $\|\nabla \vec{h}\|^{2}=\sum K_{A B C}^{2}$.)

Since

$$
\Delta h(m)=\Delta\left(\sum g^{A B} h_{A B}\right)(m)=\sum_{C}\left(\frac{\partial^{2} g^{A B}}{\partial\left(x_{C}\right)^{2}}+2 \frac{\partial g^{A B}}{\partial x_{C}} \frac{\partial h_{A B}}{\partial x_{C}}+g^{A B} \frac{\partial^{2} h_{A B}}{\partial\left(x_{C}\right)^{2}}\right)(m),
$$

we get from (2.14), (2.15) and (2.24)

$$
\Delta h(m)=\sum_{A, C} L_{A A C C}-2 \sum_{A} \lambda_{A}^{3}+6\|\vec{h}\|^{2}+3 h^{2}-h\|\vec{h}\|^{2}-3(n+2) h+n(n+2) .
$$

Similarly,

$$
(\Delta \vec{h})_{A B}(m)=\sum_{C}\left(\frac{\partial^{2} h_{A B}}{\partial\left(x_{C}\right)^{2}}-\lambda_{B} \frac{\partial \Gamma_{C A}^{B}}{\partial x_{C}}-\lambda_{A} \frac{\partial \Gamma_{C B}^{A}}{\partial x_{C}}\right)(m),
$$

and hence,

$$
\begin{aligned}
\langle\Delta \vec{h}, \vec{h}\rangle(m)= & \sum_{A, C} \lambda_{A} L_{A A C C}-2 \sum_{A} \lambda_{A}^{4}+6 \sum_{A} \lambda_{A}^{3} \\
& +\left(-\|\vec{h}\|^{4}+3 h\|\vec{h}\|^{2}-2(n+3)\|\vec{h}\|^{2}-h^{2}+(n+2) h\right)(m) .
\end{aligned}
$$

\section{An integral formula for the volume of an extrinsic ball.}

Proof of Theorem 1.1 Let us express the volume of $B(m, r) \cap M$. We have, for sufficiently small $r$, 


$$
\operatorname{vol}\left(B^{n+1}(m, r) \cap M\right)=\int_{\Omega(r)} \frac{1}{z^{n}} \sqrt{D} d x_{1} \cdots d x_{n},
$$

where $\Omega(r)$ is the orthogonal projection of $B(m, r) \cap M \quad$ on $\mathbf{R}^{n} \cong\{0\} \times \mathbf{R}^{n} \subset \mathbf{R}^{n+1}$.

Let us introduce spherical coordinates in $\mathrm{R}^{n},\left(\rho, a_{1}, \cdots, a_{n}\right)$ by

$$
x_{A}=\rho a_{A}, \quad 1 \leq A \leq n ; \quad \sum_{A=1}^{n} a_{A}^{2}=1 .
$$

This means that each point $a=\left(a_{1}, \cdots, a_{n}\right)$ belongs to the unit sphere $S^{n-1}(1)=S^{n-1}$ in $\mathrm{R}^{n}$, and $\rho$ is given by (2.3).

We shall need the following lemma

Lemma. For any sufficiently small $r>0$, the domain $\Omega(r)$ is given by

$$
\Omega(r)=\{(\rho, a) \mid 0 \leq \rho<P(a, r)\},
$$

where $P(a, r)$ is the unique solution of the equation

$$
r=\log \left(z \frac{\sqrt{\left(\rho^{2}+z^{2}-1\right)^{2}+4 \rho^{2}}+\rho^{2}+z^{2}-1}{\sqrt{\left(\rho^{2}+z^{2}-1\right)^{2}+4 \rho^{2}}-\rho^{2}+z^{2}-1}\right),
$$

or equivalently,

$$
\cosh r=\frac{z^{2}+\rho^{2}+1}{2 z}
$$

Proof. The set $\Omega(r)$ is a sublevel set of the function

$$
x \mapsto \frac{z(x)^{2}+\rho^{2}+1}{2 z(x)}
$$

where $\rho=\left(\sum_{A=1}^{n} x_{A}^{2}\right)^{1 / 2}$. The result follows from the fact that, in a neighbourhood of the origin, this function is strictly convex, since

$$
\frac{\partial^{2}}{\partial x_{A} \partial x_{B}}\left(\frac{z^{2}+\rho^{2}+1}{2 z}\right)=\delta_{i j}+O\left(\rho^{2}\right) .
$$

Using the spherical coordinates and the above lemma, we can transform the integral in (3.1) to the form

$$
\operatorname{vol}\left(B^{n+1}(m, r) \cap M\right)=\int_{S^{n-1}} d \sigma\left(\int_{0}^{P(a, r)} \frac{1}{z^{n}} \sqrt{D\left(\rho, a_{1}, \cdots, a_{n}\right)} \rho^{n-1} d \rho\right)
$$


where $d \sigma$ denotes the volume element on $S^{n-1}$. In particular, we shall denote

$$
\omega_{n-1}=\int_{S^{n-1}} d \sigma=\text { the }(n-1) \text {-dimensional volume of } S^{n-1} .
$$

Near the origin, we can write

$$
z=1+\lambda \rho^{2}+K \rho^{3}+L \rho^{4}+O\left(\rho^{5}\right),
$$

where, according to (2.2),

$\lambda=\frac{1}{2} \sum\left(\lambda_{A}-1\right) a_{A}^{2} ; \quad K=\frac{1}{6} \sum K_{A B C} a_{A} a_{B} a_{C} ; \quad L=\frac{1}{24} \sum L_{A B C D} a_{A} a_{B} a_{C} a_{D}$.

Since

$$
z_{A}^{\prime}=\left(\lambda_{A}-1\right) x_{A}+\frac{1}{2} \sum_{B, C} K_{A B C} x_{B} x_{C}+\frac{1}{6} \sum_{B, C, D} L_{A B C D} x_{B} x_{C} x_{D}+O\left(\rho^{5}\right),
$$

we have that

$$
\frac{1}{2} \sum_{A} z_{A}^{\prime 2}=\bar{B} \rho^{2}+\bar{K} \rho^{3}+\bar{L} \rho^{4}+O\left(\rho^{5}\right)
$$

where

$$
\begin{gathered}
\bar{B}=\frac{1}{2} \sum_{A}\left(\lambda_{A}-1\right)^{2} a_{A}^{2} ; \quad \bar{K}=\frac{1}{2} \sum\left(\lambda_{A}-1\right) K_{A B C} a_{A} a_{B} a_{C} ; \\
\bar{L}=\frac{1}{6} \sum\left(\lambda_{A}-1\right) L_{A B C D}+\frac{1}{8} \sum_{A}\left(\sum_{B, C} K_{A B C} a_{B} a_{C}\right)^{2} .
\end{gathered}
$$

Thus,

$$
\begin{aligned}
\sqrt{D(\rho, a)} & =\left(1+\sum_{A} z_{A}^{\prime 2}\right)^{1 / 2}=1+\frac{1}{2}\left(\sum_{A} z_{A}^{\prime 2}\right)^{2}-\frac{1}{8}\left(\sum_{A} z_{A}^{\prime 2}\right)^{4}+O\left(\rho^{5}\right) \\
& =1+\bar{B} \rho^{2}+\bar{K} \rho^{3}+\left(\bar{L}-\frac{1}{2} \bar{B}^{2}\right) \rho^{4}+O\left(\rho^{5}\right) .
\end{aligned}
$$

On the other hand,

$$
\frac{1}{z^{n}}=1-n \lambda \rho^{2}-n K \rho^{3}+\left(-n L-\frac{n(n-1) \lambda^{2}}{2}+n^{2} \lambda^{2}\right) \rho^{4}+O\left(\rho^{5}\right) .
$$

Hence our integral (3.4) takes the form 
$\operatorname{vol}\left(B^{n+1}(m, r) \cap M\right)=\int_{S^{n-1}} d \sigma\left(\int_{0}^{P(a, r)}\left(\rho^{n-1}+(\bar{B}-n \lambda) \rho^{n+1}+(\bar{K}-n K) \rho^{n+2}\right.\right.$

$$
\begin{aligned}
& \left.\left.+\left(\bar{L}-\frac{1}{2} \bar{B}^{2}-n \lambda \bar{B}+\frac{n(n+1)}{2} \lambda^{2}-n L\right) \rho^{n+3}+O\left(\rho^{n+4}\right)\right)\right) d \rho \\
& =\int_{S^{n-1}} d \sigma\left[\frac{\rho^{n}}{n}+\frac{(\bar{B}-n \lambda) \rho^{n+2}}{n+2}+\frac{(\bar{K}-n K) \rho^{n+3}}{n+3}\right. \\
& \left.+\left(\bar{L}-\frac{1}{2} \bar{B}^{2}-n \lambda \bar{B}+\frac{n(n+1)}{2} \lambda^{2}-n L\right) \frac{\rho^{n+4}}{n+4}+O\left(\rho^{n+5}\right)\right]_{\rho=0}^{\rho=P(a, r)} .
\end{aligned}
$$

We shall now estimate the bound $P(a, r)$. For any fixed $a=\left(a_{1}, \cdots, a_{n}\right) \in S^{n-1}$, and any small $r>0$, the solution $\rho=P(a, r)$ of (3.3) is given by

$$
\begin{aligned}
& \rho=r+\frac{1}{24}\left(1+12 \lambda-12 \lambda^{2}\right) r^{3}+\frac{1}{2} K(1-2 \lambda) r^{4} \\
& +\frac{1}{1920}\left(1-960 K^{2}+960 L+120 \lambda-1920 L \lambda+600 \lambda^{2}-2400 \lambda^{3}+1680 \lambda^{4}\right) r^{5}+\mathrm{O}\left(r^{6}\right) .
\end{aligned}
$$

Substituting (3.6) in (3.5) we get

$$
\begin{aligned}
& \operatorname{vol}\left(B^{n+1}(m, r) \cap M\right)=\int_{S^{n-1}}\left[\frac{r^{n}}{n}+\left(\frac{1}{24}+\frac{1}{2} \lambda-\frac{1}{2} \lambda^{2}+\frac{\bar{B}-n \lambda}{n+2}\right) r^{n+2}\right. \\
& +\left(\frac{1}{2} K-K \lambda+\frac{\bar{K}-n K}{n+3}\right) r^{n+3}+\left(\frac{5 n-2}{5760}-\frac{1}{2} K^{2}-\frac{n-2}{48} \lambda+\frac{5 n^{2}-42 n+40}{48(n+4)} \lambda^{2}\right. \\
& \quad+\frac{n-4}{4} \lambda^{3}+\frac{n+6}{8} \lambda^{4}-L \lambda+\frac{1}{24} \bar{B}-\frac{n-4}{2(n+4)} \lambda \bar{B}-\frac{1}{2} \lambda^{2} \bar{B}-\frac{1}{2(n+4)} \bar{B}^{2} \\
& \left.\left.\quad+\frac{1}{n+4} \bar{L}+\frac{4-n}{2(n+4)} L\right) r^{n+4}+\mathrm{O}\left(r^{n+5}\right)\right] d \sigma .
\end{aligned}
$$

Now we compute this integral term by term. If $\omega_{n-1}$ denotes de volume of the unit sphere in $\mathrm{R}^{n}$, and $\alpha_{n}=\omega_{n-1} / n$, then taking account of the values for the integrals over $S^{n-1}$ of the monomials in the coordinate functions ([KP, page 125$]$ ), we have, by a routine computation,

$$
\begin{aligned}
& \int_{S^{n-1}} \frac{r^{n}}{n} d \sigma=\frac{1}{n} \omega_{n-1} r^{n}=\alpha_{n} r^{n}, \quad \int_{S^{n-1}} \frac{1}{24} r^{n+2} d \sigma=\frac{n}{24} \alpha_{n} r^{n+2}, \\
& \int_{S^{n-1}} \frac{1}{2} \lambda r^{n+2} d \sigma=\frac{1}{4}(h-n) \alpha_{n} r^{n+2}, \\
& \int_{S^{n-1}}-\frac{1}{2} \lambda^{2} r^{n+2} d \sigma=-\frac{\alpha_{n} r^{n+2}}{8(n+2)}\left(2\|\vec{h}\|^{2}+h^{2}-2(n+2) h+n(n+2)\right), \\
& \int_{S^{n-1}} \frac{\bar{B}-\lambda n}{n+2} r^{n+2} d \sigma=\frac{\alpha_{n} r^{n+2}}{2(n+2)}\left(\|\vec{h}\|^{2}-(n+2) h+n(n+1)\right) .
\end{aligned}
$$


Then,

$\int_{S^{n-1}}\left(\frac{1}{24}+\frac{1}{2} \lambda-\frac{1}{2} \lambda^{2}+\frac{\bar{B}-n \lambda}{n+2}\right) r^{n+2} d \sigma=\frac{\alpha_{n} r^{n+2}}{24(n+2)}\left(6\|\vec{h}\|^{2}-3 h^{2}+4 n(n-1)\right)$.

On the other hand,

$$
\int_{S^{n-1}}\left(\frac{1}{2} K-K \lambda+\frac{\bar{K}-n K}{n+3}\right) r^{n+3} d \sigma=0,
$$

since the coefficient of $r^{n+3}$ involves only terms of odd degree in the spherical coordinates.

Finally,

$$
\begin{aligned}
& \int_{S^{n-1}} \lambda r^{n+4} d \sigma=\frac{\alpha_{n} r^{n+4}}{2}(h-n), \\
& \int_{S^{n-1}} \lambda^{2} r^{n+4} d \sigma=\frac{\alpha_{n} r^{n+4}}{4(n+2)}\left(2\|\vec{h}\|^{2}+h^{2}-2(n+2) h+n(n+2)\right), \\
& \int_{S^{n-1}} \lambda^{3} r^{n+4} d \sigma=\frac{\alpha_{n} r^{n+4}}{8(n+4)(n+2)}\left(8 \sum_{A} \lambda_{A}^{3}+6 h\|\vec{h}\|^{2}-6(n+4)\|\vec{h}\|^{2}+h^{3}\right. \\
& \left.-3(n+4) h^{2}+3(n+4)(n+2) h-n(n+2)(n+4)\right) \text {, } \\
& \int_{S^{n-1}} \lambda^{4} r^{n+4} d \sigma=\frac{\alpha_{n} r^{n+4}}{16(n+6)(n+4)(n+2)}\left(48 \sum_{A} \lambda_{A}^{4}+32 h \sum_{A} \lambda_{A}^{3}-32(n+6) \sum_{A} \lambda_{A}^{3}\right. \\
& -24(n+6) h\|\vec{h}\|^{2}+12(n+4)(n+6)\|\vec{h}\|^{2}-4(n+2)(n+4)(n+6) h \\
& +n(n+2)(n+4)(n+6)+6(n+4)(n+6) h^{2}+12\|\vec{h}\|^{4}+12 h^{2}\|\vec{h}\|^{2} \\
& \left.-4(n+6) h^{3}+h^{4}\right) \\
& \int_{S^{n-1}} \bar{B} r^{n+4} d \sigma=\frac{\alpha_{n} r^{n+4}}{2}\left(\|\vec{h}\|^{2}-2 h+n\right), \\
& \int_{S^{n-1}} \lambda \bar{B} r^{n+4} d \sigma=\frac{\alpha_{n} r^{n+4}}{4(n+2)}\left(2 \sum_{A} \lambda_{A}^{3}+h\|\vec{h}\|^{2}-(n+6)\|\vec{h}\|^{2}\right. \\
& \left.-2 h^{2}+3(n+2) h-n(n+2)\right) \text {, } \\
& \int_{S^{n-1}} \lambda^{2} \bar{B} r^{n+4} d \sigma=\frac{\alpha_{n} r^{n+4}}{8(n+4)(n+2)}\left(8 \sum_{A} \lambda_{A}^{4}+4 h \sum_{A} \lambda_{A}^{3}-4(n+8) \sum_{A} \lambda_{A}^{3}+2\|\vec{h}\|^{4}\right. \\
& +h^{2}\|\vec{h}\|^{2}-2(n+10) h\|\vec{h}\|^{2}+(n+4)(n+12)\|\vec{h}\|^{2}-2 h^{3} \\
& \left.+5(n+4) h^{2}-4(n+2)(n+4) h+n(n+2)(n+4)\right) \text {, }
\end{aligned}
$$


ON THE VOLUME OF A SMALl EXTRINSIC BALL IN A HYPERSURFACE ... 231

$$
\begin{gathered}
\int_{S^{n-1}} \bar{B}^{2} r^{n+4} d \sigma=\frac{\alpha_{n} r^{n+4}}{4(n+2)}\left(2 \sum_{A} \lambda_{A}^{4}-8 \sum_{A} \lambda_{A}^{3}+\|\vec{h}\|^{4}-4 h\|\vec{h}\|^{2}+2(n+6)\|\vec{h}\|^{2}\right. \\
\left.+4 h^{2}-4(n+2) h+n(n+2)\right),
\end{gathered}
$$

and

$$
\begin{aligned}
\int_{S^{n-1}} & \left(-\frac{1}{2} K^{2}-L \lambda+\frac{1}{n+4} \bar{L}+\frac{4-n}{2(n+4)} L\right) r^{n+4} d \sigma \\
= & \frac{\alpha_{n} r^{n+4}}{144(n+4)(n+2)}\left(24 \sum_{A, B, C} K_{A B C}^{2}+36 \sum_{A, B} \lambda_{A} L_{A A B B}-9 h \sum_{A, B} L_{A A B B}\right) .
\end{aligned}
$$

It follows that the integral of the term in $r^{n+4}$ is equal to

$$
\begin{aligned}
& \frac{\alpha_{n} r^{n+4}}{5760(n+2)(n+4)}\left(1440\langle\Delta \vec{h}, \vec{h}\rangle+960 \sum_{A, B, C} K_{A B C}^{2}-360 h \Delta h-120\left(n^{2}+2\right) h^{2}\right. \\
& +45 h^{4}+240(n-1)(n+4)\|\vec{h}\|^{2}-180 h^{2}\|\vec{h}\|^{2}+540\|\vec{h}\|^{4}-720 h \sum_{A} \lambda_{A}^{3} \\
& \left.+720 \sum_{A} \lambda_{A}^{4}+16 n(n-1)(n+2)(5 n-7)\right) \\
& =\frac{\alpha_{n} r^{n+4}}{5760(n+2)(n+4)}\left(1440\langle\Delta \vec{h}, \vec{h}\rangle+960\|\nabla \vec{h}\|^{2}+180\|R\|^{2}+360\|\rho\|^{2}-360 h \Delta h\right. \\
& -120\left(n^{2}-6 n+14\right) h^{2}+45 h^{4}-240\left(n^{2}+2\right)\|\vec{h}\|^{2}-540 h^{2}\|\vec{h}\|^{2}+900\|\vec{h}\|^{4} \\
& \left.+8 n(n-1)\left(10 n^{2}-39 n+62\right)\right) .
\end{aligned}
$$

This completes the proof of Theorem 1.1.

\section{Some geometric consequences.}

We compare now the volume of the extrinsic ball of radius $r$ with that of the $n$-ball of radius $r$ in $\mathrm{H}^{n}$, which coincides of course with the extrinsic ball of radius $r$ of $\mathrm{H}^{n}$ embedded as a totally geodesic submanifold of $\mathrm{H}^{n+1}$. is

From [Sa, page 308] we know that the volume of the ball of radius $r$ in $\mathrm{H}^{n}$

$$
n \alpha_{n} \int_{0}^{r} \sinh ^{n-1} u d u .
$$


The power series development of this expression is

$$
\alpha_{n}\left(r^{n}+\frac{n(n-1)}{6(n+2)} r^{n+2}+\frac{n(n-1)(5 n-7)}{360(n+4)} r^{n+4}+O\left(r^{n+6}\right)\right) .
$$

By equating the terms of the same degree in (4.1) and Theorem 1.1, we get

COROllary 4.1 Let $M$ be a hypersurface of $\mathrm{H}^{n+1}$, of class $C^{5}$, satisfying HEVC. Then we have

$$
2\|\vec{h}\|^{2}=h^{2}
$$

and

$$
\begin{gathered}
96\langle\Delta \vec{h}, \vec{h}\rangle+64\|\nabla \vec{h}\|^{2}-12\|R\|^{2}+24\|\rho\|^{2}-24 h \Delta h-8\left(n^{2}-6 n+14\right) h^{2} \\
(4.3)+3 h^{4}+16\left(n^{2}+2\right)\|\vec{h}\|^{2}-36 h^{2}\|\vec{h}\|^{2}+60\|\vec{h}\|^{4}-24 n(n-1)(n-2)=0 .
\end{gathered}
$$

If $M$ is of class $C^{3}$ and satisfies $\mathrm{HEVC}$, then it still satisfies (4.2).

Proof of Proposition 1.2 a) is trivial, since for $n=1$, condition (4.2) implies that $M$ is totally geodesic. For $n=2$, this condition means that

$$
2\left(\lambda_{1}^{2}+\lambda_{2}^{2}\right)=\left(\lambda_{1}+\lambda_{2}\right)^{2},
$$

which clearly implies $\lambda_{1}=\lambda_{2}$. Therefore, $M$ must be a totally umbilical submanifold. According to [S], a totally umbilical hypersurface of the hyperbolic space must be either of the following:

1) A totally geodesic hypersurface,

2) A geodesic sphere,

3) A horospheres or

4) An equidistant hypersurface.

A straightforward computation shows that each of the above hypersurfaces satisfies HEVC. In the cases 1), 3) and 4) this condition is satisfied for extrinsic balls of any radius.

Proposition 1.3 follows directly from Corollary 4.1 and (2.22).

Finally,

Proof of Proposition 1.4 From $\nabla \vec{h}=0$, and equations (4.2) and (4.3), we get

$$
24\langle\Delta \vec{h}, \vec{h}\rangle-3\|R\|^{2}+6\|\rho\|^{2}+12(n-2) h^{2}-6 n(n-1)(n-2)=0 .
$$

From the identities

$$
2\langle\Delta \vec{h}, \vec{h}\rangle=\Delta\left(\|\vec{h}\|^{2}\right)-2\|\nabla \vec{h}\|^{2}
$$


and

$$
\Delta\left(\|\vec{h}\|^{2}\right)=\frac{1}{2} \Delta\left(h^{2}\right)=h \Delta h+\|\operatorname{grad} h\|^{2},
$$

along with $\nabla \vec{h}=0$, we get that $\langle\Delta \vec{h}, \vec{h}\rangle=0$. Besides, for $n=3$, the Weyl component of the curvature tensor vanishes, which yields $\|R\|^{2}-4\|\rho\|^{2}+\tau^{2}=0$. So, for $n=3$, under our hypotheses, (4.4) becomes

$$
-12-2\|\rho\|^{2}+\tau^{2}+4 h^{2}=0 .
$$

From (2.19), we have that

$$
\|\rho\|^{2}=\left(\lambda_{1} \lambda_{3}+\lambda_{1} \lambda_{2}-2\right)^{2}+\left(\lambda_{1} \lambda_{2}+\lambda_{2} \lambda_{3}-2\right)^{2}+\left(\lambda_{1} \lambda_{3}+\lambda_{2} \lambda_{3}-2\right)^{2}
$$

and

$$
\tau=2\left(\lambda_{1} \lambda_{2}+\lambda_{1} \lambda_{3}+\lambda_{2} \lambda_{3}-3\right) .
$$

Thus, (4.5) is equivalent to

$$
h\left(h+2 \lambda_{1} \lambda_{2} \lambda_{3}\right)=0 .
$$

Then either $h=0$, which implies $\|\vec{h}\|=0$, and $M$ is totally geodesic, or $h+2 \lambda_{1} \lambda_{2} \lambda_{3}=0$. But this condition also implies that $M$ is totally geodesic. In fact, from

$$
\lambda_{1}+\lambda_{2}+\lambda_{3}+2 \lambda_{1} \lambda_{2} \lambda_{3}=0
$$

it follows that the principal curvatures cannot be all positive nor all negative. If any of them vanishes, then $h=0$, whence $\vec{h}=0$. If one of them, say $\lambda_{3}$, is negative, and the other two, $\lambda_{1}, \lambda_{2}$ are positive, then, from (4.2) we have

$$
2\left(\lambda_{1}^{2}+\lambda_{2}^{2}+\lambda_{3}^{2}\right)=\left(\lambda_{1}+\lambda_{2}+\lambda_{3}\right)^{2} .
$$

which can be written as

$$
\left(\lambda_{1}-\lambda_{2}\right)^{2}+\lambda_{3}^{2}=2 \lambda_{3}\left(\lambda_{1}+\lambda_{2}\right),
$$

and this is impossible, for the left-hand side is positive and the right-hand side is negative. The impossibility of having one of the $\lambda$ 's positive and the other two negative follows in the same way. Thus, (4.6) implies $h=0$, and hence, $M$ is totally geodesic. 


\section{REFERENCES}

[GV] A. Gray and L. Vanhecke, Riemannian geometry as determined by the volumes of small geodesic balls, Acta Math. 142 (1979), 157-198.

[KaP] L. Karp and M. Pinsky, Volume of a small extrinsic ball in a submanifold, Bull. London Math. Soc. 21 (1989), 87-92.

[KN] S. Kobayashi and K. Nomizu, Foundations of Differential Geometry, Interscience, 1963, 1969.

[KP] O. Kowalski and D. Preiss, Besicovitch-type properties of measures and submanifolds, J. Reine Angew. Math. 379 (1987), 115-151.

[Sa] L. Santaló, Integral Geometry and Geometric Probability, Addison-Wesley, Reading, MA, 1976.

[S] M. Spivak, Differential Geometry, Publish or Perish, 1970.

DEPARTAMENTO DE GEOMETRÍA Y TOPOLOGÍA

UNIVERSIDAD DE VALENCIA

BURJASOT (VALENCIA)

SPAIN

E-mail address: carreras@uv.es, naveira@uv.es 